\title{
OPTIMAL EMBEDDINGS OF CALDERÓN SPACES IN HÖLDER-ZYGMUND SPACES
}

\author{
ZIA BASHIR, FERNANDO COBOS and GEORGI E. KARADZHOV*
}

\begin{abstract}
We prove optimal embeddings of Calderón spaces built-up over function spaces defined in $\mathbf{R}^{n}$ with the Lebesgue measure into generalized Hölder-Zygmund spaces in the super-critical and critical cases.
\end{abstract}

\section{Introduction}

During the last thirty years many authors have investigated the problem of optimal embeddings of Sobolev type spaces and of Besov type spaces. We refer, for example, to the monograph by Nikol'skij [38] and the papers by Hansson [24], Netrusov [37], Goldman [20], Kolyada [27], Edmunds and Triebel [16], Cwikel and Pustylnik [12], [13], Edmunds, Kerman and Pick [15], Maly and Pick [30], Milman and Pustylnik [35], Cianchi [9] or the more recent papers [23], [31], [26], [32], [18], [34], [33] and [1].

Goldman and Kerman established in [22] optimal embeddings of Calderón spaces $\Lambda(E, F)$ in rearrangement-invariant spaces (see also [19]). Precise definitions of these spaces and related terminology is given in Sections 2 and 3 below. Spaces $\Lambda(E, F)$ include, among others, classical Besov spaces $B_{p, q}^{s}$ with $s>0$ and Besov spaces with generalized smoothness (see [40] and [10], [11]). Results of Goldman and Kerman refer to the sub-critical and critical cases. Here we continue their investigation dealing with the super-critical case. In our setting, the natural target space is not a rearrangement-invariant space but a generalized Hölder-Zygmund space $\mathscr{C} H$. Given a quasi-concave function $\varphi$ with $\alpha_{\varphi}=\beta_{\varphi}>0$, we consider any rearrangement-invariant space $E$ whose fundamental function $\varphi_{E}$ satisfies $\varphi_{E}(t) \approx \varphi(t), 0<t<2$. Among other results, we characterize the embedding $\Lambda(E, F) \hookrightarrow \mathscr{C} H$ by means of boundedness of the operator of Hardy type $R_{\varphi} g(t)=\int_{0}^{t} \frac{g(u)}{\varphi(u)} \frac{d u}{u}$.

${ }^{*}$ F. Cobos has been supported in part by the Spanish Ministerio de Ciencia e Innovación (MTM2010-15814). G. E. Karadzhov was partially supported by IMI-Instituto de Matemática Interdisciplinar de la UCM (Programa de Investigadores Visitantes) and by the Abdus Salam School of Mathematical Sciences, GC University Lahore as well as by a grant from HEC, Pakistan.

Received 28 November 2011, in final form 27 August 2012. 
Previous results on this problem are due to Gogatishvili, Neves and Opic [17] and Bashir and Karadzhov [3]. Paper [17] deals with optimal embeddings of Bessel potential spaces, while paper [3] with embeddings of generalized (inhomogeneous) Besov spaces $B^{k}(E, F)$. Although spaces $B^{k}(E, F)$ include Calderón spaces, results of [3] require more conditions on the spaces involved than those we need here. In fact, spaces $B^{k}(E, F)$ are defined by using the modulus of continuity of order $k$ and conditions on this parameter appear in the results of [3]. However, in our approach, the modulus of continuity is just a tool. In each case, we can choose $k$ sufficiently large so that no extra assumption is needed. Besides, even though we use some ideas originated in [22], [18] and [3], our techniques are different from those papers and also from [17].

The plan of the paper is as follows. In Section 2 we review some basic notions on function spaces. Calderón spaces and Hölder-Zygmund spaces are introduced in Section 3. Section 4 is devoted to embeddings between those spaces in the super-critical and critical cases. Finally, in Section 5, working in an ample class of quasi-norms, given a domain (respectively, target) quasinorm, we show the optimal target (respectively, domain) quasi-norm. We also show there some concrete examples where the optimal quasi-norms can be computed easily.

Results of this paper have been announced without proofs in [2].

\section{Preliminaries}

We use the notations $a_{1} \lesssim a_{2}$ or $a_{2} \gtrsim a_{1}$ for nonnegative functions or functionals to mean that the quotient $a_{1} / a_{2}$ is bounded; also, $a_{1} \approx a_{2}$ means that $a_{1} \lesssim a_{2}$ and $a_{1} \gtrsim a_{2}$. We say that $a_{1}$ is equivalent to $a_{2}$ if $a_{1} \approx a_{2}$.

Let $\varphi$ be a nonnegative function defined on $[0, \infty)$ which is quasi-concave. This means that $\varphi(t)$ is increasing (i.e. non decreasing) and $\varphi(t) / t$ is decreasing. Clearly, $\varphi(t) \leq \varphi(2 t) \leq 2 \varphi(t), t>0$. The dilation function $h_{\varphi}$ generated by $\varphi$ is defined by

$$
h_{\varphi}(u)=\sup _{0<t<\infty} \frac{\varphi(t u)}{\varphi(t)} .
$$

The function $h_{\varphi}$ is quasi-concave, sub-multiplicative and satisfies that

$$
h_{\varphi}(1)=1, \quad 1 \leq h_{\varphi}(u) h_{\varphi}(1 / u), \quad h_{\varphi}(u) \leq \max (1, u) .
$$

Therefore the lower and upper Boyd indices $\alpha_{\varphi}, \beta_{\varphi}$, defined by

$$
\alpha_{\varphi}=\sup _{0<t<1} \frac{\log h_{\varphi}(t)}{\log t} \quad \text { and } \quad \beta_{\varphi}=\inf _{1<t<\infty} \frac{\log h_{\varphi}(t)}{\log t},
$$


satisfy that $0 \leq \alpha_{\varphi} \leq \beta_{\varphi} \leq 1$. As is well-known certain integrability properties of $\varphi$ can be expressed through inequalities using the indices (see [4], p. 147).

We denote by $L_{\mathrm{loc}}$ the space of all locally integrable functions $f$ on $\mathbf{R}^{n}$ with the Lebesgue $n$-measure $|\cdot|_{n}$. Given $f \in L_{\mathrm{loc}}$, we write $f^{*}$ for its decreasing rearrangement

$$
f^{*}(t)=\inf \left\{\lambda>0:\left|\left\{x \in \mathbf{R}^{n}:|f(x)|>\lambda\right\}\right|_{n} \leq t\right\}, \quad t>0 .
$$

By $\mathbf{M}^{+}$we designate the space of all nonnegative functions $g$ which are locally integrable on $(0, \infty)$ with the Lebesgue measure. A positive function $b \in \mathbf{M}^{+}$is said to be slowly varying on $(1, \infty)$ if for all $\varepsilon>0$ the function $t^{\varepsilon} b(t)$ is equivalent to an increasing function, and the function $t^{-\varepsilon} b(t)$ is equivalent to a decreasing function. By symmetry, we say that $b$ is slowly varying on $(0,1)$ if the function $t \mapsto b\left(\frac{1}{t}\right)$ is slowly varying on $(1, \infty)$. Finally, $b$ is slowly varying if it is slowly varying on $(0,1)$ and $(1, \infty)$. We refer to [14] for details on slowly varying functions. As in the case of a quasi-concave function, we put

$$
h_{b}(u)=\sup _{0<t<\infty} \frac{b(t u)}{b(t)} .
$$

Using that $b$ is slowly varying, it is easy to check that

$$
\alpha_{b}=\sup _{0<t<1} \frac{\log h_{b}(t)}{\log t}=0=\inf _{1<t<\infty} \frac{\log h_{b}(t)}{\log t}=\beta_{b} .
$$

Let $\rho_{F}$ be a quasi-norm defined on $\mathbf{M}^{+}$with values in $[0, \infty]$ and which is monotone, in the sense that $g_{1} \leq g_{2}$ implies $\rho_{F}\left(g_{1}\right) \leq \rho_{F}\left(g_{2}\right)$. Denote by $F$ the quasi-normed space consisting of all locally integrable functions in $(0, \infty)$ with the Lebesgue measure such that $\|g\|_{F}=\rho_{F}(|g|)<\infty$.

There is an equivalent quasi-norm $\rho_{p}$, called a $p$-norm, which depends only on the space $F$ and which satisfies the triangle inequality $\rho_{p}^{p}\left(g_{1}+g_{2}\right) \leq$ $\rho_{p}^{p}\left(g_{1}\right)+\rho_{p}^{p}\left(g_{2}\right)$ for some $p \in(0,1]$ (see [28] and [5]). Subsequently, we shall denote the equivalent $p$-norm also by $\rho$. This, however, will not produce any confusion.

We say that the quasi-norm $\rho_{F}$ satisfies Minkowski's inequality if (for the equivalent $p$-norm) it holds

$$
\rho_{F}^{p}\left(\sum g_{j}\right) \lesssim \sum \rho_{F}^{p}\left(g_{j}\right), \quad g_{j} \in \mathbf{M}^{+} .
$$

If this is the case and $g, f, h \in \mathbf{M}^{+}$with $f(2 u) \approx f(u)$ and $h$ increases, then

$$
\rho_{F}^{p}\left(\int_{1}^{\infty} g(t) h(u t) f(u) \frac{d u}{u}\right) \lesssim \int_{1}^{\infty} \rho_{F}^{p}(g(t) h(u t) f(u)) \frac{d u}{u} .
$$


Indeed,

$$
\begin{aligned}
\rho_{F}^{p}\left(\int_{1}^{\infty} g(t) h(u t) f(u) \frac{d u}{u}\right) & =\rho_{F}^{p}\left(\sum_{m=0}^{\infty} \int_{2^{m}}^{2^{m+1}} g(t) h(u t) f(u) \frac{d u}{u}\right) \\
& \lesssim \rho_{F}^{p}\left(\sum_{m=0}^{\infty} g(t) h\left(2^{m+1} t\right) \int_{2^{m}}^{2^{m+1}} f(u) \frac{d u}{u}\right) \\
& \lesssim \sum_{m=0}^{\infty} \rho_{F}^{p}\left(g(t) h\left(2^{m+1} t\right)\right) \int_{2^{m+1}}^{2^{m+2}} f(u) \frac{d u}{u} \\
& \leq \sum_{m=0}^{\infty} \int_{2^{m+1}}^{2^{m+2}} \rho_{F}^{p}(g(t) h(u t) f(u)) \frac{d u}{u} \\
& \leq \int_{1}^{\infty} \rho_{F}^{p}(g(t) h(u t) f(u)) \frac{d u}{u} .
\end{aligned}
$$

Similarly,

$$
\begin{aligned}
\rho_{F}^{p}\left(\int_{0}^{1} g(t) h(u t) f(u) \frac{d u}{u}\right) \lesssim \rho_{F}^{p}(g(t) h(t)) & \int_{1 / 2}^{1} f(u) \frac{d u}{u} \\
& +\int_{0}^{1} \rho_{F}^{p}(g(t) h(u t) f(u)) \frac{d u}{u} .
\end{aligned}
$$

We shall work with the classes of functions

$$
\begin{aligned}
& N_{1}:=\left\{g \in \mathbf{M}^{+}: \operatorname{tg}(t) \text { is increasing }\right\}, \\
& N_{0}:=\left\{g \in \mathbf{M}^{+}: g(t) \text { is increasing }\right\} .
\end{aligned}
$$

The dilation function generated by $\rho_{F}$ is given by

$$
h_{F}(u)=\sup \left\{\frac{\rho_{F}\left(g_{u}\right)}{\rho_{F}(g)}: g \in N_{1}\right\} \text {. }
$$

Here $g_{u}(t)=g(t u)$.

The function $h_{F}(u)$ is sub-multiplicative with

$$
h_{F}(1)=1 \quad \text { and } \quad h_{F}(u) h_{F}(1 / u) \geq 1 .
$$

We assume that $h_{F}(u)$ is always finite. The function $h_{F}(u)$ does not need to be increasing but $u h_{F}(u)$ does, being also sub-multiplicative. So, we can proceed with $u h_{F}(u)$ to define the the Boyd indices of $F$ similarly as it is done 
in the case of a rearrangement-invariant space (see [4, Definition 3.5.2]). The resulting numbers can then be expressed only in terms of $h_{F}$. Namely, we put

$$
\alpha_{F}=\sup _{0<t<1} \frac{\log h_{F}(t)}{\log t} \quad \text { and } \quad \beta_{F}=\inf _{1<t<\infty} \frac{\log h_{F}(t)}{\log t} .
$$

These numbers satisfy that $-1 \leq \alpha_{F} \leq \beta_{F}$. In what follows, we suppose that $\alpha_{F}=\beta_{F}$.

In our further considerations, an important role will be played be the quasinormed function space $F=L_{*}^{q}\left(b(t) t^{-s / n}\right)$. Here $s \geq 0$ and $b$ is a slowly varying function, and $L_{*}^{q}(w)$, or simply $L_{*}^{q}$ if $w=1$, is the weighted Lebesgue space with a quasi-norm

$$
\|g\|_{L_{*}^{q}(w)}=\left(\int_{0}^{\infty}[w(t)|g(t)|]^{q} \frac{d t}{t}\right)^{1 / q}, \quad 0<q \leq \infty, w>0, w \in \mathbf{M}^{+} .
$$

The space $F=L_{*}^{q}\left(b(t) t^{-s / n}\right)$ is $p$-normed for $p=\min (q, 1)$. As for its Boyd indices, we have that

$$
\begin{aligned}
\left\|g_{u}\right\|_{F} & =\left(\int_{0}^{\infty}\left[b(t) t^{-s / n}|g(u t)|\right]^{q} \frac{d t}{t}\right)^{1 / q} \\
& =\left(\int_{0}^{\infty}\left[b\left(u^{-1} s\right)\left(u^{-1} s\right)^{-s / n}|g(s)|\right]^{q} \frac{d s}{s}\right)^{1 / q} \\
& \leq h_{b}\left(u^{-1}\right) u^{s / n}\|g\|_{F} .
\end{aligned}
$$

Hence, $h_{F}(u) \leq h_{b}\left(u^{-1}\right) u^{s / n}$. It follows that

$$
\beta_{F} \leq \inf _{1<u<\infty} \frac{\log h_{b}\left(u^{-1}\right)}{\log u}+\frac{s}{n}=\frac{s}{n}
$$

and

$$
\alpha_{F} \geq \sup _{0<u<1} \frac{\log h_{b}\left(u^{-1}\right)}{\log u}+\frac{s}{n}=\frac{s}{n}
$$

where we have used (2.1) in the last two equalities. Since always $\alpha_{F} \leq \beta_{F}$, we conclude that $\alpha_{F}=\beta_{F}=s / n$.

We shall also work with rearrangement-invariant Banach function spaces $E$ on $\mathbf{R}^{n}$ as defined in [4]. In particular, $\|f\|_{E}=\rho_{E}\left(f^{*}\right)$, where $\rho_{E}$ is a monotone norm satisfying Minkowski's inequality. The fundamental function $\varphi_{E}$ of $E$ is given by $\varphi_{E}(t)=\rho_{E}\left(\chi_{(0, t)}\right)$. The function $\varphi_{E}$ is quasi-concave (see [4], [29]). 


\section{Calderón spaces and Hölder-Zygmund spaces}

Subsequently, let $\varphi$ be a quasi-concave function with $\alpha_{\varphi}=\beta_{\varphi}>0$ and let $E$ be any rearrangement-invariant space whose fundamental function $\varphi_{E}$ satisfies that $\varphi_{E}(t) \approx \varphi(t), 0<t<2$. For example, $E$ can be the Marcinkiewicz space $M_{\varphi}$, normed by

$$
\|f\|_{M_{\varphi}}=\sup _{0<t<\infty} \frac{\varphi(t)}{t} \int_{0}^{t} f^{*}(s) d s,
$$

(see [4], [29]). If $\varphi(t)=t^{1 / p}$ with $1 \leq p<\infty$ then we can also choose $L^{p}$. If $1<p<\infty$, another possible choice for $E$ is the Lorentz space $L^{p, q}$. We can even choose spaces with $\alpha_{E} \neq \beta_{E}$. This is the case of $E=L^{p, q}+L^{\infty}$ whose fundamental function is $\varphi_{E}(t)=\min \left\{t^{1 / p}, 1\right\}$. Note that

$$
E \hookrightarrow E+L^{\infty} \hookrightarrow M_{\varphi_{E}}+L^{\infty}=M_{\varphi}+L^{\infty} .
$$

The last equality in (3.1) holds because $K\left(1, f ; E, L^{\infty}\right) \approx \rho_{E}\left(\chi_{(0,1)} f^{*}\right)$. Here $K$ is the Peetre's functional (see [5], [7]). Hence

$$
\begin{aligned}
\|f\|_{M_{\varphi_{E}}+L^{\infty}} & \approx \sup _{0<t<1} \frac{\varphi_{E}(t)}{t} \int_{0}^{t} f^{*}(s) d s \approx \sup _{0<t<1} \frac{\varphi(t)}{t} \int_{0}^{t} f^{*}(s) d s \\
& \approx\|f\|_{M_{\varphi}+L^{\infty}} .
\end{aligned}
$$

More examples for $E$ can be given by using the spaces

$$
\Gamma^{q}(\omega)=\left\{f:\|f\|_{\Gamma^{q}(\omega)}=\left(\int_{0}^{\infty}\left(\frac{\omega(t)}{t} \int_{0}^{t} f^{*}(s) d s\right)^{q} \frac{d t}{t}\right)^{1 / q}<\infty\right\},
$$

where $1 \leq q<\infty$.

Here the positive weight $\omega$ satisfies that $\omega(t) \in L_{*}^{q}(\min (1,1 / t))$. Note that

$$
\varphi_{\Gamma^{q}(\omega)}=\left(\int_{0}^{\infty}[\omega(u) \min (1, t / u)]^{q} \frac{d u}{u}\right)^{1 / q} .
$$

Definition 3.1. We denote by $\Lambda(E, F)$ the (inhomogeneous) Calderón space, formed by all functions $f \in E$ having a finite quasi-norm

$$
\|f\|_{\Lambda(E, F)}=\rho_{F}\left(d_{E}(t, f)\right)+\|f\|_{E} .
$$

Here

$$
d_{E}(t, f)=\inf \left\{\|f-e\|_{E}: e \in M\left(t^{-1 / n}\right)\right\}, \quad t>0,
$$

is the best approximation of $f$ by the subset $M\left(t^{-1 / n}\right) \subset E$, consisting of all functions in $E$, such that the support of their Fourier transform is containing in the cube centered at the origin and with side length $2 t^{-1 / n}$. 
The Calderón spaces have been studied in [8], [22], [21], [19] and the papers mentioned in these references.

We note that if

$$
\rho_{F}\left(\chi_{(1, \infty)}\right)<\infty
$$

then

$$
\|f\|_{\Lambda(E, F)} \approx \rho_{F}\left(\chi_{(0,1)}(t) d_{E}(t, f)\right)+\|f\|_{E},
$$

and $M(1) \subset \Lambda(E, F)$. We suppose that $\rho_{F}\left(\chi_{(0,1)}\right)=\infty$ otherwise $\Lambda(E, F)=$ E.

Calderón spaces include classical Besov spaces $B_{r, q}^{s}$ with $s>0$. Namely, $B_{r, q}^{s}=\Lambda\left(L^{r}, L_{*}^{q}\left(t^{-s / n}\right)\right)$. It is well-known that the embedding properties of Besov spaces depend on the relationship between $s$ and $n / r$. Three different cases can be considered: $s<n / r$ (sub-critical case), $s=n / r$ (critical case) and $s>n / r$ (super-critical case). Next we extend two of these concepts to our setting.

Definition 3.2. A property on Calderón spaces $\Lambda(E, F)$ with $\varphi_{E}(t) \approx$ $\varphi(t), 0<t<2$, is said to refer to the super-critical (respectively, critical) case provided that $\alpha_{F}>\alpha_{\varphi}$ (respectively, $\alpha_{F}=\alpha_{\varphi}$ ).

As we said in the Introduction, our aim is to establish optimal embeddings of Calderón spaces into generalized Hölder-Zygmund spaces $\mathscr{C} H$. In order to introduce this class of spaces, we denote the modulus of continuity of order $k$ by

$$
\omega^{k}(t, f)=\sup _{|h| \leq t} \sup _{x \in \mathbf{R}^{n}}\left|\Delta_{h}^{k} f(x)\right|,
$$

where $\Delta_{h}^{k} f$ are the usual iterated differences of $f$. When $k=1$ we simply write $\omega(t, f)$.

Let $\rho_{H}$ be a monotone quasi-norm which satisfies Minkowski's inequality. We define the dilation function $h_{H}(u)$ associated to $\rho_{H}$ by

$$
h_{H}(u)=\sup \left\{\frac{\rho_{H}\left(g_{u}\right)}{\rho_{H}(g)}: g \in N_{0}\right\} .
$$

Using the function $h_{H}$, Boyd indices of $H$ are defined as for the case of $F$. They are always non negative. We assume that $\alpha_{H}=\beta_{H}$. Sometimes it will be useful to suppose that

$$
\rho_{H}\left(\chi_{(2, \infty)}\right)<\infty
$$


Definition 3.3. Let $j=0,1, \ldots$ and let $\mathscr{C}^{j}$ stand for the space of all functions $f$, defined in $\mathbf{R}^{n}$, that have bounded and uniformly continuous derivatives up to the order $j$, normed by $\|f\|_{\mathscr{C}^{j}}=\sup \sum_{l=0}^{j}\left|P^{l} f(x)\right|$, where $P^{l} f(x)=\sum_{|v|=l} D^{v} f(x)$.

- If $j / n<\alpha_{H}<(j+1) / n$ for $j \geq 1$ or $0 \leq \alpha_{H}<1 / n$ for $j=0$, then $\mathscr{C} H$ is formed by all functions $f$ in $\mathscr{C}^{j}$ having a finite quasi-norm

$$
\|f\|_{\mathscr{C} H}=\sum_{l=0}^{j}\left\|P^{l} f\right\|_{L^{\infty}}+\rho_{H}\left(t^{j / n} \omega\left(t^{1 / n}, P^{j} f\right)\right) .
$$

- If $\alpha_{H}=(j+1) / n$, then $\mathscr{C} H$ consists of all functions $f$ in $\mathscr{C}^{j}$ having a finite quasi-norm

$$
\|f\|_{\mathscr{C} H}=\sum_{l=0}^{j}\left\|P^{l} f\right\|_{L^{\infty}}+\rho_{H}\left(t^{j / n} \omega^{2}\left(t^{1 / n}, P^{j} f\right)\right) .
$$

The following result was established in [3]. It shows an equivalent quasinorm in the Hölder-Zygmund space, which does not involve the derivatives of the function but the modulus of continuity of order $k$. We give a more direct proof than the one provided in [3].

Theorem 3.4. Assume that $\rho_{H}$ satisfies (3.4) and let $k \in \mathrm{N}$ such that $0 \leq \alpha_{H}=\beta_{H}<k / n$. Then

$$
\|f\|_{\mathscr{C} H} \approx\|f\|_{\mathscr{C}^{0}}+\rho_{H}\left(\omega^{k}\left(t^{1 / n}, f\right)\right) .
$$

Proof. The right-hand term in (3.5) is bounded by the left-hand term because $\omega^{k}\left(t^{1 / n}, f\right) \lesssim t^{j / n} \omega\left(t^{1 / n}, P^{j} f\right)$. With the aim of checking the converse inequality, let $f \in \mathscr{C}^{0}$ and let $j>0$ with $\alpha_{H}>j / n$. We have

$$
\int_{0}^{\infty} u^{-j / n} \omega^{k}\left(u^{1 / n}, f\right) \frac{d u}{u} \lesssim \int_{0}^{1} u^{-j / n} \omega^{k}\left(u^{1 / n}, f\right) \frac{d u}{u}+\|f\|_{\mathscr{C}^{0}} .
$$

For $g \in N_{0}$, we get $g(t) \rho_{H}\left(\chi_{(2, \infty)}\right) \leq \rho_{H}(g(u t)) \leq h_{H}(t) \rho_{H}(g)$. Using that $\alpha_{H}>j / n$, we obtain

$$
\begin{aligned}
\int_{0}^{1} u^{-j / n} \omega^{k}\left(u^{1 / n}, f\right) \frac{d u}{u} & \lesssim \int_{0}^{1} u^{-j / n} h_{H}(u) \frac{d u}{u} \rho_{H}\left(\omega^{k}\left(t^{1 / n}, f\right)\right) \\
& \lesssim \rho_{H}\left(\omega^{k}\left(t^{1 / n}, f\right)\right) .
\end{aligned}
$$


Hence the integral $\int_{0}^{\infty} u^{-j / n} \omega^{k}\left(u^{1 / n}, f\right) d u / u$ is convergent and then with the same arguments as in [4, Thm. 5.4.14], it follows that $f$ has derivatives up to order $j$, and moreover,

$$
\left\|P^{j} f\right\|_{L^{\infty}} \lesssim \rho_{H}\left(\omega^{k}\left(u^{1 / n}, f\right)\right)+\|f\|_{\mathscr{C}^{0}} .
$$

Analogously for $P^{l} f, l<j$.

Let $v$ be any multi-index of nonnegative integers such that $|v| \leq j$. Next we show that $D^{v} f$ is uniformly continuous. We consider the case $|\nu|=j$, the others being similar. By Marchaud's inequality (see [4, Thm. 5.4.4]), we have

$$
\omega\left(t^{1 / n}, D^{v} f\right) \lesssim t^{1 / n} \int_{t}^{\infty} u^{-1 / n} \omega^{k}\left(u^{1 / n}, D^{v} f\right) \frac{d u}{u} .
$$

Using also the estimate (cf. [4], p. 342)

$$
\omega^{k}\left(t^{1 / n}, D^{v} f\right) \lesssim \int_{0}^{t} u^{-j / n} \omega^{k}\left(u^{1 / n}, f\right) \frac{d u}{u},
$$

and Fubini's theorem, we get $\omega\left(t^{1 / n}, D^{\alpha} f\right) \lesssim A(t)$, where

$$
A(t)=t^{1 / n} \int_{t}^{\infty} u^{-(j+1) / n} \omega^{k}\left(u^{1 / n}, f\right) \frac{d u}{u}+\int_{0}^{t} u^{-j / n} \omega^{k}\left(u^{1 / n}, f\right) \frac{d u}{u}
$$

Note that $\int_{1}^{\infty} u^{-(j+1) / n} \omega^{k}\left(u^{1 / n}, f\right) \frac{d u}{u} \lesssim\|f\|_{\mathscr{C}^{0}}<\infty$. Let $0<t<1$ and put

$$
h(t)=t^{1 / n} \int_{t}^{1} u^{-(j+1) / n} \omega^{k}\left(u^{1 / n}, f\right) \frac{d u}{u} .
$$

Since $\int_{0}^{1} h(t) \frac{d t}{t} \lesssim \int_{0}^{1} u^{-j / n} \omega^{k}\left(u^{1 / n}, f\right) \frac{d u}{u}<\infty$, it follows $\lim _{t \rightarrow 0} h(t)=0$. This yields that $\lim _{t \rightarrow 0} A(t)=0$, and therefore it establishes the uniform continuity of $D^{v} f$. Hence $f \in \mathscr{C}^{j}$.

It remains to estimate $\|f\|_{\mathscr{C} H}$. Assume that $j / n<\alpha_{H}<(j+1) / n$. The other cases are analogous. Relationship between $\alpha_{H}$ and $j$ gives that

$$
\int_{0}^{1} h_{H}^{p}(u) u^{-p j / n} \frac{d u}{u}+\int_{1}^{\infty} h_{H}^{p}(u) u^{-p(j+1) / n} \frac{d u}{u}<\infty .
$$

Since $t^{j / n} \omega\left(t^{1 / n}, D^{v} f\right) \lesssim t^{j / n} A(t)$, applying Minkowski's inequality and using (3.6), we conclude that

$$
\rho_{H}\left(t^{j / n} \omega\left(t^{1 / n}, P^{j} f\right)\right) \lesssim \rho_{H}\left(\omega^{k}\left(t^{1 / n}, f\right)\right) .
$$

This completes the proof. 
Note that Theorem 3.4 yields also that

$$
\|f\|_{\mathscr{C} H} \approx\|f\|_{\mathscr{C}^{0}}+\rho_{H}\left(\chi_{(0,2)}(t) \omega^{k}\left(t^{1 / n}, f\right)\right) .
$$

As an example, let $\rho_{H}(g)=\sup t^{-\gamma / n} b(t) g(t)$, where $0 \leq \gamma \leq k$, and $b$ is slowly varying. Then $\alpha_{H}=\beta_{H}=\gamma / n$. If $0<\gamma<k$ and $b=1$ then $\mathscr{C} H$ coincide with the usual Hölder-Zygmund space $C^{\gamma}$ (see [42], [41]).

\section{Embeddings}

This section contains the more important results of the paper. We start with a preliminary lemma.

Lemma 4.1. Let $E$ be a rearrangement-invariant space. If $f \in E$ then

$$
\|f\|_{L^{\infty}} \lesssim \int_{0}^{t} \frac{d_{E}(u, f)}{\varphi_{E}(u)} \frac{d u}{u}+\frac{\|f\|_{E}}{\varphi_{E}(t)}, \quad t>0 .
$$

Proof. For integer $j$ we can find $e_{j} \in M\left(2^{-j / n}\right)$ such that $\left\|f-e_{j}\right\|_{E} \leq$ $2 d_{E}\left(2^{j}, f\right)$. Let $u_{j}=e_{j+1}-e_{j}$. Then $u_{j} \in M\left(2^{-j / n}\right)$ and for $k>m$ we have $\sum_{j=m}^{k} u_{j}=e_{k+1}-e_{m}$. Using the well-known estimate in different metrics (see [6]) we have

$$
\begin{aligned}
\left\|e_{k+1}-e_{m}\right\|_{L^{\infty}} & \leq \sum_{j=m}^{k}\left\|u_{j}\right\|_{L^{\infty}} \lesssim \sum_{j=m}^{k} \frac{\left\|u_{j}\right\|_{E}}{\varphi_{E}\left(2^{j}\right)} \\
& \lesssim \sum_{j=m}^{k} \frac{d_{E}\left(2^{j+1}, f\right)}{\varphi_{E}\left(2^{j}\right)} \lesssim \sum_{j=m}^{k+1} \frac{d_{E}\left(2^{j}, f\right)}{\varphi_{E}\left(2^{j}\right)}
\end{aligned}
$$

where we have used that $\varphi_{E}\left(2^{j+1}\right) \leq 2 \varphi_{E}\left(2^{j}\right)$. On the other hand,

$$
\begin{aligned}
\left(f-e_{m}+e_{k+1}\right)^{*}\left(2^{m+1}\right) & \leq\left(f-e_{m}\right)^{*}\left(2^{m}\right)+\left(e_{k+1}\right)^{*}\left(2^{m}\right) \\
& \lesssim \frac{\left\|f-e_{m}\right\|_{E}}{\varphi_{E}\left(2^{m}\right)}+\left\|e_{k+1}\right\|_{L^{\infty}} .
\end{aligned}
$$

Since

$$
\left\|e_{k+1}\right\|_{L^{\infty}} \lesssim \frac{\left\|e_{k+1}\right\|_{E}}{\varphi_{E}\left(2^{k+1}\right)} \lesssim \frac{\left\|f-e_{k+1}\right\|_{E}}{\varphi_{E}\left(2^{k+1}\right)}+\frac{\|f\|_{E}}{\varphi_{E}\left(2^{k+1}\right)},
$$

it follows

$$
\begin{aligned}
f^{*}\left(2^{m+2}\right) & \lesssim\left(f-e_{m}+e_{k+1}\right)^{*}\left(2^{m+1}\right)+\left\|e_{k+1}-e_{m}\right\|_{L^{\infty}} \\
& \lesssim \sum_{j=m}^{k+1} \frac{d_{E}\left(2^{j}, f\right)}{\varphi_{E}\left(2^{j}\right)}+\frac{\|f\|_{E}}{\varphi_{E}\left(2^{k+1}\right)}
\end{aligned}
$$


Letting $m \rightarrow-\infty$ we get

$$
f^{*}(0) \lesssim \sum_{j=-\infty}^{k+1} \frac{d_{E}\left(2^{j}, f\right)}{\varphi_{E}\left(2^{j}\right)}+\frac{\|f\|_{E}}{\varphi_{E}\left(2^{k+1}\right)} .
$$

Given any $0<t<\infty$, we can find $k \in \mathbf{Z}$ such that $2^{k+2}<t \leq 2^{k+3}$. Then using monotonicity of $d_{E}(t, f)$ and the fact that $\varphi_{E}$ is quasi-concave, we derive (4.1) from (4.2).

Next we discuss the embedding of $\Lambda(E, F)$ in $\mathscr{C}^{0}$. For $N \subseteq \mathbf{M}^{+}$, we consider the condition

$$
\int_{0}^{2} \frac{g(u)}{\varphi(u)} \frac{d u}{u} \lesssim \rho_{F}(g), \quad g \in N .
$$

THEOREM 4.2. Let $E$ be a rearrangement-invariant space with $\varphi_{E}(t) \approx \varphi(t)$ for $0<t<2$.

If (4.3) holds with $N=N_{0}$, then $\Lambda(E, F) \hookrightarrow \mathscr{C}^{0}$. Conversely, if $\alpha_{F}>0$ and the embedding $\Lambda(E, F) \hookrightarrow \mathscr{C}^{0}$ holds, then (4.3) is satisfied with $N=N_{1}$.

Furthermore, if we are in the super-critical case $\alpha_{F}>\alpha_{\varphi}$, then condition (4.3) is satisfied with $N=N_{1}$.

Proof. Using (4.1) and (4.3) with $N=N_{0}$, for any $f \in \Lambda(E, F)$ we obtain

$$
\|f\|_{L^{\infty}} \lesssim \int_{0}^{2} \frac{d_{E}(u, f)}{\varphi_{E}(u)} \frac{d u}{u}+\frac{\|f\|_{E}}{\varphi_{E}(2)} \lesssim \rho_{F}\left(d_{E}(u, f)\right)+\|f\|_{E}=\|f\|_{\Lambda(E, F)} .
$$

In order to check that $\lim _{t \rightarrow 0} \omega\left(t^{1 / n}, f\right)=0$, we first show that for any $k \in \mathrm{N}$

$$
\omega^{k}\left(t^{1 / n}, f\right) \lesssim \int_{0}^{t} \frac{d_{E}(u, f)}{\varphi_{E}(u)} \frac{d u}{u}+t^{k / n} \int_{t}^{\infty} u^{-k / n} \frac{d_{E}(u, f)}{\varphi_{E}(u)} \frac{d u}{u} .
$$

Take any integer $m \geq 1$ and let $\omega_{E}^{m}(t, f)=\sup _{|h| \leq t}\left\|\Delta_{h}^{m} f\right\|_{E}$. Using (4.1) and $d_{E}\left(u, \Delta_{h}^{m} f\right) \lesssim d_{E}(u, f)$, we derive for $|h| \leq t^{1 / n}$,

$$
\left|\Delta_{h}^{m} f(x)\right| \lesssim \int_{0}^{t} \frac{d_{E}(u, f)}{\varphi_{E}(u)} \frac{d u}{u}+\frac{\omega_{E}^{m}\left(t^{1 / n}, f\right)}{\varphi_{E}(t)} .
$$

Since by the inverse theorem of approximation theory (see [22], [39]),

$$
\omega_{E}^{m}\left(t^{1 / n}, f\right) \lesssim \int_{0}^{t} d_{E}(u, f) \frac{d u}{u}+t^{m / n} \int_{t}^{\infty} u^{-m / n} d_{E}(u, f) \frac{d u}{u},
$$


we obtain

$$
\omega^{m}\left(t^{1 / n}, f\right) \lesssim \int_{0}^{t} \frac{d_{E}(u, f)}{\varphi_{E}(u)} \frac{d u}{u}+\frac{t^{m / n}}{\varphi_{E}(t)} \int_{t}^{\infty} u^{-m / n} d_{E}(u, f) \frac{d u}{u} .
$$

By Marchaud's inequality,

$$
\omega^{k}\left(t^{1 / n}, f\right) \lesssim t^{k / n} \int_{t}^{\infty} u^{-k / n} \omega^{m}\left(u^{1 / n}, f\right) \frac{d u}{u}, \quad k<m .
$$

Hence using (4.5) and Fubini's theorem, we have

$$
\begin{aligned}
\omega^{k}\left(t^{1 / n}, f\right) \lesssim \int_{0}^{t} \frac{d_{E}(u, f)}{\varphi_{E}(u)} \frac{d u}{u}+t^{k / n} \int_{t}^{\infty} u^{-k / n} \frac{d_{E}(u, f)}{\varphi_{E}(u)} \frac{d u}{u} \\
+t^{k / n} \int_{t}^{\infty} u^{-m / n} d_{E}(u, f) \int_{0}^{u} \frac{v^{(m-k) / n}}{\varphi_{E}(v)} \frac{d v}{v} \frac{d u}{u} .
\end{aligned}
$$

The choice of $m$ such that $\beta_{\varphi_{E}}<(m-k) / n$ yields $\int_{0}^{u} \frac{v^{(m-k) / n}}{\varphi_{E}(v)} \frac{d v}{v} \lesssim \frac{u^{(m-k) / n}}{\varphi_{E}(u)}$, thus (4.4) is proved.

Now we proceed with the limit of $\omega\left(t^{1 / n}, f\right)$. Writing down (4.4) with $k=1$, we get

$$
\omega\left(t^{1 / n}, f\right) \lesssim \int_{0}^{t} \frac{d_{E}(u, f)}{\varphi_{E}(u)} \frac{d u}{u}+t^{1 / n} \int_{t}^{\infty} \frac{u^{-1 / n} d_{E}(u, f)}{\varphi_{E}(u)} \frac{d u}{u} .
$$

Let $0<t<1$. By (4.3) we obtain

$$
\int_{0}^{t} \frac{d_{E}(u, f)}{\varphi_{E}(u)} \frac{d u}{u} \leq \int_{0}^{1} \frac{d_{E}(u, f)}{\varphi_{E}(u)} \frac{d u}{u} \lesssim \rho_{F}\left(\chi_{(0,1)}(u) d_{E}(u, f)\right) \lesssim\|f\|_{\Lambda(E, F)} .
$$

Therefore, using the dominated convergence theorem, we conclude that

$$
\lim _{t \rightarrow 0} \int_{0}^{t} \frac{d_{E}(u, f)}{\varphi_{E}(u)} \frac{d u}{u}=0 .
$$

As for the second term in (4.6), first note that

$$
\int_{1}^{\infty} \frac{u^{-1 / n} d_{E}(u, f)}{\varphi_{E}(u)} \frac{d u}{u} \lesssim\|f\|_{E} \int_{1}^{\infty} u^{-1 / n} \frac{d u}{u} \lesssim\|f\|_{E} .
$$

If

$$
\int_{0}^{\infty} \frac{u^{-1 / n} d_{E}(u, f)}{\varphi_{E}(u)} \frac{d u}{u}<\infty
$$


then clearly

$$
\lim _{t \rightarrow 0} t^{1 / n} \int_{t}^{\infty} \frac{u^{-1 / n} d_{E}(u, f)}{\varphi_{E}(u)} \frac{d u}{u}=0 .
$$

If (4.7) does not hold, we proceed as follows. Since $\int_{0}^{1} \frac{d_{E}(u, f)}{\varphi_{E}(u)} \frac{d u}{u}$ is convergent, we have that

$$
\sum_{n=0}^{\infty} \frac{d_{E}\left(2^{-(n+1)}, f\right)}{\varphi_{E}\left(2^{-(n+1)}\right)} \lesssim \sum_{n=0}^{\infty} \frac{d_{E}\left(2^{-(n+1)}, f\right)}{\varphi_{E}\left(2^{-n}\right)} \lesssim \sum_{n=0}^{\infty} \int_{2^{-(n+1)}}^{2^{-n}} \frac{d_{E}(u, f)}{\varphi_{E}(u)} \frac{d u}{u}<\infty .
$$

Hence $\lim _{n \rightarrow \infty} \frac{d_{E}\left(2^{-n}, f\right)}{\varphi_{E}\left(2^{-n}\right)}=0$. This implies that $\lim _{t \rightarrow 0} \frac{d_{E}(t, f)}{\varphi_{E}(t)}=0$. Now, using l'Hopital's rule, we derive

$$
\begin{aligned}
\lim _{t \rightarrow 0} t^{1 / n} \int_{t}^{\infty} \frac{u^{-1 / n} d_{E}(u, f)}{\varphi_{E}(u)} \frac{d u}{u} & =\lim _{t \rightarrow 0} \frac{\int_{t}^{\infty} \frac{u^{-1 / n} d_{E}(u, f)}{\varphi_{E}(u)} \frac{d u}{u}}{t^{-1 / n}} \\
& =\lim _{t \rightarrow 0} \frac{d_{E}(t, f)}{\varphi_{E}(t)}=0 .
\end{aligned}
$$

Consequently, $\lim _{t \rightarrow 0} \omega\left(t^{1 / n}, f\right)=0$.

Suppose that $\alpha_{F}>0$ and that the embedding $\Lambda(E, F) \hookrightarrow \mathscr{C}^{0}$ holds. To prove the necessity of (4.3) with $N=N_{1}$, we shall construct a suitable test function $f$ as follows. Let

$$
\psi(x)=\prod_{j=1}^{n} \frac{\sin ^{2}\left(x_{j} / 2\right)}{\left(x_{j} / 2\right)^{2}} \quad \text { for } \quad x=\left(x_{1}, \ldots, x_{n}\right) \neq 0
$$

and $\psi(0)=1$. This function satisfies that $0 \leq \psi(x) \leq 1$ and $\psi(x) \leq$ $2^{2 n} /\left(x_{1}^{2} \cdots x_{n}^{2}\right)$. Moreover, (see [6], [19])

$$
\psi\left(x u^{-1 / n}\right) \in M\left(u^{-1 / n}\right) \quad \text { and } \quad\left\|\psi\left(x u^{-1 / n}\right)\right\|_{E} \lesssim \varphi_{E}(u) .
$$

Given any $g \in N_{1} \cap F$, put

$$
f(x)=\int_{0}^{2} \frac{g(u)}{\varphi(u)} \psi\left(x u^{-1 / n}\right) \frac{d u}{u} .
$$

We claim that $f$ belongs to $\Lambda(E, F)$. Indeed, for $g \in N_{1}$ we have

$$
\rho_{F}(g) \geq \rho_{F}\left(\chi_{(t, \infty)}(u) u g(u) u^{-1}\right) \geq \operatorname{tg}(t) \rho_{F}\left(\chi_{(t, \infty)}(u) u^{-1}\right)
$$

and

$$
\rho_{F}\left(\chi_{(1, \infty)}(u) u^{-1}\right) \leq t h_{F}(t) \rho_{F}\left(\chi_{(t, \infty)}(u) u^{-1}\right) .
$$


Hence

$$
g(t) \lesssim h_{F}(t) \rho_{F}(g), \quad g \in N_{1}
$$

Using this inequality and the fact that $\alpha_{F}>0$, we derive that

$$
\begin{aligned}
\|f\|_{E} & \leq \int_{0}^{2} \frac{g(u)}{\varphi(u)}\left\|\psi\left(x u^{-1 / n}\right)\right\|_{E} \frac{d u}{u} \lesssim \int_{0}^{2} g(u) \frac{d u}{u} \\
& \lesssim \rho_{F}(g) \int_{0}^{2} h_{F}(u) \frac{d u}{u} \lesssim \rho_{F}(g) .
\end{aligned}
$$

It follows that $f \in E$. Next, for $0<t<2$, write

$$
f_{0 t}(x)=\int_{0}^{t} \frac{g(u)}{\varphi(u)} \psi\left(x u^{-1 / n}\right) \frac{d u}{u}, \quad f_{1 t}(x)=\int_{t}^{2} \frac{g(u)}{\varphi(u)} \psi\left(x u^{-1 / n}\right) \frac{d u}{u} .
$$

We have $f=f_{0 t}+f_{1 t}$ with $f_{1 t} \in M\left(t^{-1 / n}\right)$. Whence,

$$
d_{E}(t, f) \leq\left\|f_{0 t}\right\|_{E} \leq \int_{0}^{t} g(u) \frac{d u}{u}, \quad 0<t<2 .
$$

Using Minkowski's inequality and that $\alpha_{F}>0$, we derive

$$
\begin{aligned}
\rho_{F}^{p}\left(\chi_{(0,2)}(t) d_{E}(t, f)\right) & \leq \rho_{F}^{p}\left(\int_{0}^{t} g(u) \frac{d u}{u}\right)=\rho_{F}^{p}\left(\int_{0}^{1} g(v t) \frac{d v}{v}\right) \\
& \lesssim \rho_{F}^{p}(g)+\int_{0}^{1} \rho_{F}^{p}(g(v t)) \frac{d v}{v} \\
& \leq \rho_{F}^{p}(g)+\left(\int_{0}^{1} h_{F}^{p}(v) \frac{d v}{v}\right) \rho_{F}^{p}(g) \\
& \lesssim \rho_{F}^{p}(g) .
\end{aligned}
$$

Therefore $f$ belongs to $\Lambda(E, F)$ and so $f \in \mathscr{C}^{0}$. Moreover, since (3.3)) also holds replacing $\chi_{(0,1)}$ by $\chi_{(0,2)}$, we have that $\|f\|_{\Lambda(E, F)} \lesssim \rho_{F}(g)$. Finally, since

$$
\int_{0}^{2} \frac{g(u)}{\varphi(u)} \frac{d u}{u}=f(0) \lesssim\|f\|_{\Lambda(E, F)} \lesssim \rho_{F}(g),
$$

inequality (4.3) with $N=N_{1}$ follows.

Suppose now that we are in the super-critical case. Using (4.9) and that $1 / \varphi(u) \lesssim h_{\varphi}(1 / u)$, we can write

$$
\int_{0}^{2} \frac{g(u)}{\varphi(u)} \frac{d u}{u} \lesssim \rho_{F}(g) \int_{0}^{2} h_{F}(u) h_{\varphi}(1 / u) \frac{d u}{u}, \quad g \in N_{1} .
$$


Then to derive (4.3) it suffices to notice that the integral in the right-hand side is convergent because $\alpha_{F}>\alpha_{\varphi}$. This completes the proof.

We use condition (4.3) in the following definition where we introduce some notation related to embeddings of Calderón spaces in Hölder-Zygmund spaces. We also introduce a new condition related to boundedness of the operator $K g=g / \varphi$.

DeFINITION 4.3. Let $E$ be a rearrangement-invariant Banach function space with $\varphi_{E}(t) \approx \varphi(t), 0<t<2$.

- We denote by $\mathcal{N}_{d}$ the collection of all monotone quasi-norms $\rho_{F}$ satisfying (3.2), Minkowski's inequality, with $\beta_{F}=\alpha_{F} \geq \beta_{\varphi}=\alpha_{\varphi}>0$ and such that

$$
\int_{0}^{2} \frac{g(u)}{\varphi(u)} \frac{d u}{u} \lesssim \rho_{F}(g), \quad g \in N_{1} .
$$

If $\rho_{F} \in \mathscr{N}_{d}$ we say that $\rho_{F}$ (respectively, $F$ ) is a domain quasi-norm (respectively, a domain space).

- The set $\mathcal{N}_{t}$ consists of all monotone quasi-norms $\rho_{H}$ which satisfy (3.4), Minkowski's inequality, and with $\alpha_{H}=\beta_{H}$. If $\rho_{H} \in \mathcal{N}_{t}$ we say that $\rho_{H}$ (respectively, $H$ ) is a target quasi-norm (respectively, a target space).

- For $j=0,1$, we put $\mathcal{N}_{j}$ for the collection of all couples $\left(\rho_{F}, \rho_{H}\right) \in$ $\mathscr{N}_{d} \times \mathcal{N}_{t}$ such that

$$
\rho_{H}\left(\chi_{(0,2)} \frac{g}{\varphi}\right) \lesssim \rho_{F}(g), \quad g \in N_{j} .
$$

- We say that the couple of quasi-norms $\left(\rho_{F}, \rho_{H}\right)$ is admissible if

$$
\Lambda(E, F) \hookrightarrow \mathscr{C} H .
$$

Note that $\mathscr{N}_{1} \subseteq \mathcal{N}_{0}$. Next we shall characterize admissible couples $\left(\rho_{F}, \rho_{H}\right)$ in the super-critical and critical cases. The Hardy type operator

$$
R_{\varphi} g(t)=\int_{0}^{t} \frac{g(u)}{\varphi(u)} \frac{d u}{u}, \quad t>0,
$$

will play an outstanding role in our considerations.

THeOREM 4.4. Let $j=0$, 1 . In the super-critical case $\alpha_{F}>\alpha_{\varphi}$, if $\left(\rho_{F}, \rho_{H}\right)$ $\in \mathscr{N}_{j}$ then for the operator $R_{\varphi}$ the following estimate holds

$$
\rho_{H}\left(\chi_{(0,2)} R_{\varphi} g\right) \lesssim \rho_{F}(g), \quad g \in N_{j} .
$$


Proof. Using Minkowski's inequality and (4.10), for any $g \in N_{j}$ we derive

$$
\begin{aligned}
& \rho_{H}^{p}\left(\chi_{(0,2)}(t) \int_{0}^{t} \frac{g(u)}{\varphi(u)} \frac{d u}{u}\right) \\
& \quad=\rho_{H}^{p}\left(\chi_{(0,2)}(t) \int_{0}^{1} \frac{g(v t) \varphi(t)}{\varphi(v t) \varphi(t)} \frac{d v}{v}\right) \\
& \quad \leq \rho_{H}^{p}\left(\int_{0}^{1} \frac{\chi_{(0,2)}(t)}{t \varphi(t)} v t g(v t) \frac{h_{\varphi}\left(v^{-1}\right)}{v} \frac{d v}{v}\right) \\
& \quad \lesssim \rho_{H}^{p}\left(\chi_{(0,2)} \frac{g}{\varphi}\right)+\int_{0}^{1} \rho_{H}^{p}\left(\chi_{(0,2)}(t) \frac{g(v t)}{\varphi(t)}\right) h_{\varphi}^{p}\left(v^{-1}\right) \frac{d v}{v} \\
& \quad \lesssim \rho_{F}^{p}(g)+\int_{0}^{1} \rho_{F}^{p}(g(v t)) h_{\varphi}^{p}\left(v^{-1}\right) \frac{d v}{v} \\
& \quad=\rho_{F}^{p}(g)\left[1+\int_{0}^{1} h_{F}^{p}(v) h_{\varphi}^{p}\left(v^{-1}\right) \frac{d v}{v}\right]
\end{aligned}
$$

and the integral is finite because $\alpha_{F}>\alpha_{\varphi}$.

Remark 4.5. Condition (4.10) is weaker than (4.11). Indeed, if $g \in N_{j}$ we have

$$
\int_{0}^{t} \frac{g(2 u)}{\varphi(u)} \frac{d u}{u} \approx \int_{0}^{2 t} \frac{g(u)}{\varphi(u)} \frac{d u}{u} \gtrsim \int_{t}^{2 t} \frac{g(u)}{\varphi(u)} \frac{d u}{u} \gtrsim \frac{g(t)}{\varphi(t)} .
$$

Whence, using (4.11), we derive that

$$
\rho_{H}\left(\chi_{(0,2)} \frac{g}{\varphi}\right) \lesssim \rho_{F}(g(2 u)) \lesssim \rho_{F}(g)
$$

The following result shows that working with quasi-norms in $\mathcal{N}_{0}$, condition (4.11) is sufficient for the embedding $\Lambda(E, F) \hookrightarrow \mathscr{C} H$.

Theorem 4.6. Assume that $\left(\rho_{F}, \rho_{H}\right) \in \mathcal{N}_{0}$ and that (4.11) is satisfied with $j=0$. Then the couple $\left(\rho_{F}, \rho_{H}\right)$ is admissible.

Proof. Take $k \in \mathrm{N}$ such that $k / n>\beta_{F}$. It follows from (4.4) that

$$
\omega^{k}\left(t^{1 / n}, f\right) \lesssim \int_{0}^{t} \frac{d_{E}(u, f)}{\varphi_{E}(u)} \frac{d u}{u}+\frac{t^{k / n}}{\varphi_{E}(t)} \int_{t}^{\infty} u^{-k / n} d_{E}(u, f) \frac{d u}{u} .
$$

Whence

$$
\begin{aligned}
\rho_{H}^{p}\left(\chi_{(0,2)}(t) \omega^{k}\left(t^{1 / n}, f\right)\right) \lesssim & \rho_{H}^{p}\left(\chi_{(0,2)}(t) R_{\varphi} d_{E}(f)(t)\right) \\
& +\rho_{H}^{p}\left(\chi_{(0,2)}(t) \frac{t^{k / n}}{\varphi(t)} \int_{t}^{\infty} u^{-k / n} d_{E}(u, f) \frac{d u}{u}\right) .
\end{aligned}
$$


Consider the two terms at the right-hand side. By (4.11), the first term is bounded from above by $\rho_{F}^{p}\left(d_{E}(t, f)\right)$. As for the second term, using Minkowski's inequality and (4.10), we derive

$$
\begin{aligned}
& \rho_{H}^{p}\left(\chi_{(0,2)}(t) \frac{t^{k / n}}{\varphi(t)} \int_{t}^{\infty} u^{-k / n} d_{E}(u, f) \frac{d u}{u}\right) \\
& \quad=\rho_{H}^{p}\left(\chi_{(0,2)}(t) \frac{1}{\varphi(t)} \int_{1}^{\infty} u^{-k / n} d_{E}(t u, f) \frac{d u}{u}\right) \\
& \quad \lesssim \int_{1}^{\infty} u^{-k p / n} \rho_{H}^{p}\left(\chi_{(0,2)}(t) \frac{d_{E}(t u, f)}{\varphi(t)}\right) \frac{d u}{u} \\
& \quad \lesssim \int_{1}^{\infty} u^{-k p / n} \rho_{F}^{p}\left(d_{E}(t u, f)\right) \frac{d u}{u} \\
& \leq\left(\int_{1}^{\infty} u^{-k p / n} h_{F}^{p}(u) \frac{d u}{u}\right) \rho_{F}^{p}\left(d_{E}(t, f)\right) .
\end{aligned}
$$

Since $k / n>\beta_{F}$, the integral is finite. According to (3.7) and Theorem 4.2, we conclude

$$
\begin{aligned}
\|f\|_{\mathscr{C} H} & \approx\|f\|_{L_{\infty}}+\rho_{H}\left(\chi_{(0,2)}(t) \omega^{k}\left(t^{1 / n}, f\right)\right) \\
& \lesssim\|f\|_{\Lambda(E, F)}+\rho_{F}\left(d_{E}(t, f)\right) \lesssim\|f\|_{\Lambda(E, F)} .
\end{aligned}
$$

The proof is finished.

The next result refers to the necessity of (4.11).

THEOREM 4.7. Let $\left(\rho_{F}, \rho_{H}\right) \in \mathscr{N}_{1}$. If $\left(\rho_{F}, \rho_{H}\right)$ is admissible, then the estimate (4.11) holds for $R_{\varphi}$ with $j=1$.

Proof. Given any $g \in N_{1} \cap F$, let $f$ be the function defined in (4.8). As we have shown in the proof of Theorem 4.2, $f$ belongs to $\Lambda(E, F)$ and so to $\mathscr{C} H$ because the couple is admissible. Moreover $\|f\|_{\Lambda(E, F)} \lesssim \rho_{F}(g)$. In order to estimate $\rho_{H}\left(\chi_{(0,2)} R_{\varphi} g\right)$, let $C>0$ to be determine later and let $k \in \mathrm{N}$ such that $\alpha_{F}-\alpha_{\varphi}<k / n$. We have

$$
\omega^{k}\left(t^{1 / n}, f\right) \approx \omega^{k}\left(C t^{1 / n}, f\right) \gtrsim \omega^{k}\left(C t^{1 / n}, f_{0 t}\right)-\omega^{k}\left(C t^{1 / n}, f_{1 t}\right) .
$$

Clearly, if $|h| \leq C t^{1 / n}$, we have $\omega^{k}\left(C t^{1 / n}, f_{0 t}\right) \geq\left|\left(\Delta_{h}^{k} f_{0 t}\right)(0)\right|$. Take $h_{j}=$ $C t^{1 / n} / \sqrt{n}$ and $h=\left(h_{1}, \ldots h_{n}\right)$. For $0<u<t<2$, since

$$
\psi\left(j h u^{-1 / n}\right) \leq \frac{2^{2 n}}{\frac{C^{2 n}}{n^{n}} t^{2} j^{2 n} u^{-2}} \leq 2^{2 n}\left(\frac{n}{C^{2}}\right)^{n},
$$


it is not hard to check that we can choose $C$ large enough such that

$$
\left|\left(\Delta_{h}^{k} f_{0, t}\right)(0)\right| \geq \frac{1}{2} \int_{0}^{t} \frac{g(u)}{\varphi(u)} \frac{d u}{u} .
$$

With this choice of $C$, we derive

$$
\omega^{k}\left(C t^{1 / n}, f_{0 t}\right) \gtrsim \frac{1}{2} \int_{0}^{t} \frac{g(u)}{\varphi(u)} \frac{d u}{u}=\frac{1}{2} R_{\varphi} g(t) .
$$

On the other hand,

$$
\omega^{k}\left(C t^{1 / n}, f_{1 t}\right) \approx \omega^{k}\left(t^{1 / n}, f_{1 t}\right) \lesssim t^{k / n}\left\|D^{k} f_{1 t}\right\|_{L_{\infty}} \lesssim t^{k / n} \int_{t}^{2} \frac{g(u)}{\varphi(u)} u^{-k / n} \frac{d u}{u} .
$$

It follows that

$$
\chi_{(0,2)}(t) R_{\varphi} g(t) \lesssim \chi_{(0,2)}(t) \omega^{k}\left(t^{1 / n}, f\right)+\chi_{(0,2)}(t) t^{k / n} \int_{t}^{2} \frac{g(u)}{\varphi(u)} u^{-k / n} \frac{d u}{u} .
$$

Applying $\rho_{H}$, we derive

$$
\begin{aligned}
\rho_{H}\left(\chi_{(0,2)} R_{\varphi} g\right) & \lesssim\|f\|_{\Lambda(E, F)}+\rho_{H}\left(\chi_{(0,2)}(t) t^{k / n} \int_{t}^{2} \frac{g(u)}{\varphi(u)} u^{-k / n} \frac{d u}{u}\right) \\
& \lesssim \rho_{F}(g)+\rho_{H}\left(\chi_{(0,2)}(t) t^{k / n} \int_{t}^{2} \frac{g(u)}{\varphi(u)} u^{-k / n} \frac{d u}{u}\right) .
\end{aligned}
$$

We proceed to estimate the last term. For $0<t<2$, a change of variable, Minkowski's inequality and (4.10) yield

$$
\begin{aligned}
& \rho_{H}^{p}\left(\chi_{(0,2)}(t) t^{k / n} \int_{t}^{2} \frac{g(u)}{\varphi(u)} u^{-k / n} \frac{d u}{u}\right) \\
& \quad \leq \rho_{H}^{p}\left(\chi_{(0,2)}(t) \int_{1}^{\infty} \frac{g(v t)}{\varphi(v t)} v^{-k / n} \frac{d v}{v}\right) \\
& \quad \lesssim \int_{1}^{\infty} \rho_{H}^{p}\left(\chi_{(0,2)}(t) \frac{g(v t)}{\varphi(v t)}\right) v^{-p k / n} \frac{d v}{v} \\
& \lesssim \int_{1}^{\infty} \rho_{H}^{p}\left(\chi_{(0,2)}(t) \frac{g(v t)}{\varphi(t)}\right) v^{-p k / n} h_{\varphi}^{p}\left(v^{-1}\right) \frac{d v}{v} \\
& \lesssim \int_{1}^{\infty} \rho_{F}^{p}(g(v t)) v^{-p k / n} h_{\varphi}^{p}\left(v^{-1}\right) \frac{d v}{v} \\
& \lesssim \rho_{F}^{p}(g) \int_{1}^{\infty} h_{F}^{p}(v) v^{-p k / n} h_{\varphi}^{p}\left(v^{-1}\right) \frac{d v}{v} .
\end{aligned}
$$


The integral is finite because $\alpha_{F}-\alpha_{\varphi}<k / n$.

Consequently, $\rho_{H}\left(\chi_{(0,2)} R_{\varphi} g\right) \lesssim \rho_{F}(g)$. The proof is completed.

As a direct consequence of the last three theorems, we obtain the following.

CoROLlary 4.8. In the super-critical case, if $\left(\rho_{F}, \rho_{H}\right) \in \mathcal{N}_{0}$ then $\left(\rho_{F}, \rho_{H}\right)$ is admissible.

Corollary 4.9. Assume that we are in the critical case. If $\left(\rho_{F}, \rho_{H}\right) \in \mathscr{N}_{0}$ and (4.11) holds with $j=0$, then $\left(\rho_{F}, \rho_{H}\right)$ is admissible. Conversely, if $\left(\rho_{F}, \rho_{H}\right) \in \mathcal{N}_{1}$ and it is admissible, then (4.11) is satisfied with $j=1$.

\section{Optimality and examples}

Subsequently, we write for simplicity $\mathscr{N}$ instead of $\mathscr{N}_{1}$. Let again $E$ be a rearrangement-invariant space with $\varphi_{E}(t) \approx \varphi(t), 0<t<2$. We start by discussing optimality of quasi-norms with respect to the embedding $\Lambda(E, F) \hookrightarrow$ $\mathscr{C} H$ in the class $\mathcal{N}$. We consider first the super-critical case.

THEOREM 5.1. Given any $\rho_{F} \in \mathcal{N}_{d}$ with $\alpha_{F}>\beta_{\varphi}>0$ and $\rho_{F}\left(\chi_{(1, \infty)} \varphi\right)<$ $\infty$, put

$$
\rho_{H(F)}(g)=\rho_{F}(\varphi g), \quad g \in \mathbf{M}^{+} .
$$

Then $\rho_{H(F)} \in \mathscr{N}_{t}$ and $\left(\rho_{F}, \rho_{H(F)}\right) \in \mathcal{N}$. Moreover $\rho_{F}$ and $\rho_{H(F)}$ are optimal in the following sense:

(i) If $\left(\rho_{F}, \rho_{H_{1}}\right)$ belongs to $\mathcal{N}$, then $\mathscr{C} H(F) \hookrightarrow \mathscr{C} H_{1}$.

(ii) If $\left(\rho_{F_{1}}, \rho_{H(F)}\right) \in \mathcal{N}$ is admissible, then $\Lambda\left(E, F_{1}\right) \hookrightarrow \Lambda(E, F)$.

Proof. Let $\rho(g)=\rho_{F}(\varphi g)$. Clearly $\rho$ is monotone, satisfies Minkowski's inequality and (3.4). Let us check that $\alpha_{\rho}=\beta_{\rho}=\alpha_{F}-\alpha_{\varphi}$. For $g \in N_{0}$, we have

$$
\rho(g(s t))=\rho_{F}(\varphi(t) g(s t)) \leq h_{\varphi}\left(s^{-1}\right) \rho_{F}(\varphi(s t) g(s t)) .
$$

Then $h_{\rho}(s) \leq h_{\varphi}\left(s^{-1}\right) h_{F}(s)$ and so

$$
\log h_{\rho}(s) \leq \log h_{F}(s)+\log h_{\varphi}\left(s^{-1}\right) .
$$

Using [4, (5.20) in page 147], we derive

$$
\beta_{\rho}=\lim _{s \rightarrow \infty} \frac{\log h_{\rho}(s)}{\log s} \leq \lim _{s \rightarrow \infty} \frac{\log h_{F}(s)}{\log s}-\lim _{t \rightarrow 0} \frac{\log h_{\varphi}(t)}{\log t}=\beta_{F}-\alpha_{\varphi} .
$$

Similarly, we obtain

$$
\alpha_{\rho}=\lim _{s \rightarrow 0} \frac{\log h_{\rho}(s)}{\log s} \geq \lim _{s \rightarrow 0} \frac{\log h_{F}(s)}{\log s}-\lim _{t \rightarrow \infty} \frac{\log h_{\varphi}(t)}{\log t}=\alpha_{F}-\beta_{\varphi} .
$$


Since $\varphi$ satisfies that $\alpha_{\varphi}=\beta_{\varphi}$ and $F$ that $\alpha_{F}=\beta_{F}$, we conclude that

$$
\alpha_{F}-\alpha_{\varphi} \leq \alpha_{\rho} \leq \beta_{\rho} \leq \alpha_{F}-\alpha_{\varphi}
$$

which implies the wanted equality.

We have that $\left(\rho_{F}, \rho\right) \in \mathcal{N}$ because

$$
\rho\left(\chi_{(0,2)} \frac{g}{\varphi}\right)=\rho_{F}\left(\chi_{(0,2)} g\right) \leq \rho_{F}(g), \quad g \in N_{1} .
$$

Since $\alpha_{F}>\alpha_{\varphi}$, the couple $\left(\rho_{F}, \rho\right)$ is admissible.

Assume that $\rho_{H_{1}} \in \mathscr{N}_{t}$ with $\left(\rho_{F}, \rho_{H_{1}}\right) \in \mathscr{N}$. Using (4.10), for any $g \in N_{1}$, we get

$$
\rho_{H_{1}}\left(\chi_{(0,2)} g\right)=\rho_{H_{1}}\left(\chi_{(0,2)} \frac{\varphi g}{\varphi}\right) \lesssim \rho_{F}(\varphi g)=\rho(g) .
$$

Therefore, by (3.7), we conclude that $\mathscr{C} H(F) \hookrightarrow \mathscr{C} H_{1}$.

Finally, if $\left(\rho_{F_{1}}, \rho\right) \in \mathscr{N}$ is an admissible couple then

$$
\rho_{F}\left(\chi_{(0,2)} g\right)=\rho\left(\chi_{(0,2)} \frac{g}{\varphi}\right) \lesssim \rho_{F_{1}}(g), \quad g \in N_{1} .
$$

Since

$$
\|f\|_{\Lambda(E, F)} \approx \rho_{F}\left(\chi_{(0,2)}(t) d_{E}(t, f)\right)+\|f\|_{E},
$$

we conclude that $\Lambda\left(E, F_{1}\right) \hookrightarrow \Lambda(E, F)$.

Next we deal with the critical case. Now the optimal target quasi-norm is not of so simple as in the previous case.

Theorem 5.2. Let $\rho_{F} \in \mathcal{N}_{d}$. Put

$$
\rho_{H(F)}(g)=\inf \left\{\rho_{F}(h): g \leq R_{\varphi} h, h \in N_{1}\right\}, \quad g \in \mathbf{M}^{+} .
$$

Then $\rho_{H(F)} \in \mathscr{N}_{t}$, the couple $\left(\rho_{F}, \rho_{H(F)}\right)$ belongs to $\mathcal{N}$ and it is admissible.

Furthermore, if $\left(\rho_{F}, \rho_{H_{1}}\right) \in \mathcal{N}$ is an admissible couple, then $\mathscr{C} H(F) \hookrightarrow$ $\mathscr{C} \mathrm{H}_{1}$.

Proof. It is clear that $\rho_{H(F)}$ is monotone and satisfies Minkowski's inequality. For $t \geq 2$,

$$
R_{\varphi} \chi_{(1, \infty)}(t)=\int_{0}^{t} \frac{\chi_{(1, \infty)}(u)}{\varphi(u)} \frac{d u}{u} \geq \int_{1}^{2} \frac{1}{\varphi(u)} \frac{d u}{u} \geq C \chi_{(2, \infty)}(t) .
$$

So, $\rho_{H(F))}\left(\chi_{(2, \infty)}\right) \leq C \rho_{F}\left(\chi_{(1, \infty)}\right)<\infty$. 
If $g \leq R_{\varphi} f$, then

$$
g(t s) \leq \int_{0}^{t} \frac{f(u s)}{\varphi(u s)} \frac{d u}{u} \leq \int_{0}^{t} h_{\varphi}\left(s^{-1}\right) \frac{f(u s)}{\varphi(u)} \frac{d u}{u} .
$$

This yields that $h_{H(F)}(s) \leq h_{F}(s) h_{\varphi}(1 / s)$. Now proceeding as we did in the proof of Theorem 5.1 to compute the indices of $\rho$, it follows that $\alpha_{H(F)}=$ $\beta_{H(F)}=\alpha_{F}-\alpha_{\varphi}$.

To check (4.3), take any $g \in N_{1}$. We have

$$
\int_{0}^{t} \frac{g(2 u)}{\varphi(u)} \frac{d u}{u} \approx \int_{0}^{2 t} \frac{g(u)}{\varphi(u)} \frac{d u}{u} \gtrsim \int_{t}^{2 t} \frac{g(u)}{\varphi(u)} \frac{d u}{u} \gtrsim \frac{g(t)}{\varphi(t)} .
$$

Therefore

$$
\rho_{H(F)}\left(\chi_{(0,2)} \frac{g}{\varphi}\right) \lesssim \rho_{F}(g(2 u)) \lesssim \rho_{F}(g) .
$$

Since (4.11) is clearly satisfied with $j=1$, Theorem 4.6 implies that the couple $\left(\rho_{F}, \rho_{H(F)}\right)$ is admissible. Now suppose that the couple $\left(\rho_{F}, \rho_{H_{1}}\right)$ belongs to $\mathcal{N}$ and it is admissible. We are going to show that

$$
\rho_{H_{1}}\left(\chi_{(0,2)} g\right) \leq \rho_{H(F)}\left(\chi_{(0,2)} g\right), \quad g \in N_{1} \text {. }
$$

Then, according to (3.7), the embedding $\mathscr{C} H(F) \hookrightarrow \mathscr{C} H_{1}$ will follow.

Take any $h \in N_{1}$ such that $\chi_{(0,2)} g \leq R_{\varphi} h$. We have

$\chi_{(0,2)}(t) g(t) \leq \chi_{(0,2)}(t) \int_{0}^{t} \chi_{(0,2)}(u) \frac{h(u)}{\varphi(u)} \frac{d u}{u}+\chi_{(0,2)}(t) \int_{0}^{t} \chi_{(2, \infty)}(u) \frac{h(u)}{\varphi(u)} \frac{d u}{u}$.

The last integral cancels out, so $\chi_{(0,2)} g \leq \chi_{(0,2)} R_{\varphi}\left(\chi_{(0,2)} h\right)$. Using Theorem 4.7, we obtain $\rho_{H_{1}}\left(\chi_{(0,2)} g\right) \leq \rho_{H_{1}}\left(\chi_{(0,2)} R_{\varphi}\left(\chi_{(0,2)} h\right)\right) \lesssim \rho_{F}(h)$. This implies (5.1) and completes the proof.

Now we turn our attention to the optimal domain quasi-norm of a given target quasi-norm.

THEOREM 5.3. Let $\rho_{H} \in \mathcal{N}_{t}$ such that

$$
\rho_{H}\left(\chi_{(1, \infty)}(t) \int_{1}^{t} \frac{1}{\varphi(u)} \frac{d u}{u}\right)<\infty
$$

Put

$$
\rho_{F(H)}(g)=\rho_{H}\left(R_{\varphi} g\right), \quad g \in \mathbf{M}^{+} .
$$

Then $\rho_{F(H)} \in \mathcal{N}_{d},\left(\rho_{F(H)}, \rho_{H}\right) \in \mathcal{N}$ and it is an admissible couple. 
Furthermore:

(i) If $\left(\rho_{F_{1}}, \rho_{H}\right) \in \mathscr{N}$ and it is admissible, then $\Lambda\left(E, F_{1}\right) \hookrightarrow \Lambda(E, F(H))$.

(ii) If $\left(\rho_{F(H)}, \rho_{H_{1}}\right) \in \mathcal{N}$ is admissible, then $\mathscr{C} H \hookrightarrow \mathscr{C} H_{1}$.

Proof. It is clear that $\rho_{F(H)}$ is monotone and satisfies Minkowski's inequality. Since $h_{F(H)}(u) \leq h_{\varphi}(u) h_{H}(u)$, it follows that $\alpha_{F(H)}=\beta_{F(H)}=\alpha_{H}+\alpha_{\varphi}$. We also have

$$
\rho_{F(H)}\left(\chi_{(1, \infty)}\right)=\rho_{H}\left(R_{\varphi} \chi_{(1, \infty)}\right)=\rho_{H}\left(\chi_{(1, \infty)}(t) \int_{1}^{t} \frac{1}{\varphi(u)} \frac{d u}{u}\right)<\infty .
$$

For $g \in N_{1}$, we get

$$
\begin{aligned}
\rho_{F(H)}(g) & =\rho_{H}\left(\int_{0}^{t} \frac{g(u)}{\varphi(u)} \frac{d u}{u}\right) \geq \rho_{H}\left(\chi_{(2, \infty)}(t) \int_{0}^{t} \frac{g(u)}{\varphi(u)} \frac{d u}{u}\right) \\
& \geq \rho_{H}\left(\chi_{(2, \infty)}\right) \int_{0}^{2} \frac{g(u)}{\varphi(u)} \frac{d u}{u} \gtrsim \int_{0}^{2} \frac{g(u)}{\varphi(u)} \frac{d u}{u} .
\end{aligned}
$$

Hence, $\rho_{F(H)}$ belongs to $\mathscr{N}_{d}$.

In order to check (4.10), take any $g \in N_{1}$. We have

$$
\begin{aligned}
\rho_{F(H))}(g) & \approx \rho_{F(H))}(g(2 u))=\rho_{H}\left(\int_{0}^{t} \frac{g(2 u)}{\varphi(u)} \frac{d u}{u}\right) \gtrsim \rho_{H}\left(\int_{t / 2}^{t} \frac{g(2 u)}{\varphi(u)} \frac{d u}{u}\right) \\
& \gtrsim \rho_{H}\left(\frac{g(t)}{\varphi(t)} \int_{t / 2}^{t} \frac{d u}{u}\right) \gtrsim \rho_{H}\left(\frac{g(t)}{\varphi(t)}\right) \gtrsim \rho_{H}\left(\chi_{(0,2)} \frac{g}{\varphi}\right) .
\end{aligned}
$$

The couple $\left(\rho_{F(H)}, \rho_{H}\right)$ is admissible because (4.11) is clearly satisfied with $j=1$. As for the optimality, if $\left(\rho_{F_{1}}, \rho_{H}\right)$ belongs to $\mathcal{N}$ and it is admissible, then

$$
\rho_{H}\left(\chi_{(0,2)} \frac{g}{\varphi}\right) \lesssim \rho_{F_{1}}(g) \quad \text { for } \quad g \in N_{1},
$$

and $R_{\varphi}$ satisfies the bounded condition (4.11) between $\rho_{F_{1}}$ and $\rho_{H}$. It follows that

$$
\begin{aligned}
\rho_{F(H)}\left(\chi_{(0,2)} g\right) & \lesssim \rho_{H}\left(\chi_{(0,2)} R_{\varphi}\left(\chi_{(0,2)} g\right)\right)+\rho_{H}\left(\chi_{(2, \infty)} R_{\varphi}\left(\chi_{(0,2)} g\right)\right) \\
& \lesssim \rho_{F_{1}}(g)+\rho_{H}\left(\chi_{(2, \infty)} R_{\varphi}\left(\chi_{(0,2)} g\right)\right) .
\end{aligned}
$$

To estimate the $\rho_{H}$ quasi-norm, note that for $t>2$, we obtain

$$
\begin{aligned}
\int_{0}^{t} \frac{\chi_{(0,2)}(u) g(u)}{\varphi(u)} \frac{d u}{u} & =\int_{0}^{2} \frac{\chi_{(0,2)}(u) g(u)}{\varphi(u)} \frac{d u}{u}+\int_{2}^{\infty} \frac{\chi_{(0,2)}(u) g(u)}{\varphi(u)} \frac{d u}{u} \\
& =\int_{0}^{2} \frac{g(u)}{\varphi(u)} \frac{d u}{u} \lesssim \rho_{F_{1}}(g)
\end{aligned}
$$


where we have used (4.3) in the last inequality. Whence,

$$
\rho_{H}\left(\chi_{(2, \infty)} R_{\varphi}\left(\chi_{(0,2)} g\right)\right) \lesssim \rho_{H}\left(\chi_{(2, \infty)}\right) \rho_{F_{1}}(g) \lesssim \rho_{F_{1}}(g) .
$$

It follows that

$$
\rho_{F(H)}\left(\chi_{(0,2)} g\right) \lesssim \rho_{F_{1}}(g), \quad g \in N_{1} .
$$

Since (3.3) still holds replacing $\chi_{(0,1)}$ by $\chi_{(0,2)}$, we derive the continuous embedding $\Lambda\left(E, F_{1}\right) \hookrightarrow \Lambda(E, F(H))$.

Finally, assume that $\left(\rho_{F(H)}, \rho_{H_{1}}\right) \in \mathcal{N}$ is an admissible couple and let us show that $\mathscr{C} H \hookrightarrow \mathscr{C} H_{1}$. First note that if $f \in \mathscr{C} H$ then $\omega^{k}\left(t^{1 / n}, f\right)$ is increasing, with $\omega^{k}\left((2 t)^{1 / n}, f\right) \leq 2^{k} \omega^{k}\left(t^{1 / n}, f\right)$ and $\lim _{t \rightarrow 0} \omega^{k}\left(t^{1 / n}, f\right)=0$. Let $g$ be any function in $N_{0}$ such that $g(2 t) \leq 2^{k} g(t)$ and $\lim _{t \rightarrow 0} g(t)=0$. According to (3.7), to establish the embedding between the Hölder-Zygmund spaces, it suffices to show that there is $C>0$ independent of $g$ such that

$$
\rho_{H_{1}}\left(\chi_{(0,2)} g\right) \leq C \rho_{H}(g) .
$$

Put

$$
h(t)=\frac{1}{t} \int_{0}^{t} g(u) d u=\int_{0}^{1} g(t v) d v .
$$

The function $h$ is increasing, with

$$
h(t) \leq g(t) \leq 2^{k+1} \frac{1}{t} g\left(\frac{t}{2}\right) \frac{t}{2} \leq 2^{k+1} \frac{1}{t} \int_{t / 2}^{t} g(u) d u \leq 2^{k+1} h(t) .
$$

Hence, (5.3) follows if

$$
\rho_{H_{1}}\left(\chi_{(0,2)} h\right) \leq \rho_{H}(h)
$$

holds. Moreover, in view of (5.1), to prove (5.4) it is enough to check that

$$
\rho_{H(F(H))}\left(\chi_{(0,2)} h\right) \leq \rho_{H}(h) .
$$

Let $h_{1}(t)=t h^{\prime}(t)=g(t)-h(t) \geq 0$. Since

$$
t h_{1}(t)=\operatorname{tg}(t)-\int_{0}^{t} g(u) d u=\int_{0}^{t}(g(t)-g(u)) d u,
$$

we have that $h_{1} \in N_{1}$ and therefore $\varphi_{E} h_{1} \in N_{1}$. Moreover, $R_{\varphi}\left(\varphi h_{1}\right)=h$. Consequently, using Theorem 5.2, we conclude

$$
\rho_{H(F(H))}\left(\chi_{(0,2)} h\right) \leq \rho_{H(F(H))}(h) \leq \rho_{F(H)}\left(\varphi h_{1}\right)=\rho_{H}\left(R_{\varphi}\left(\varphi h_{1}\right)\right)=\rho_{H}(h) .
$$

This shows (5.5) and finishes the proof. 
Next we give examples where the optimal quasi-norms can be computed explicitly. We consider similar situations to those studied in [3] for generalized Besov spaces.

ExAmPLe 5.4. Let $H=L_{*}^{1}(v)$, satisfying (5.2) and with $\alpha_{H}=\beta_{H}$. Then (3.4) holds. Put $w(t)=\int_{t}^{\infty} v(u) d u / u$. According to Theorem 5.3, the optimal domain quasi-norm is

$$
\rho_{F}(g)=\rho_{H}\left(R_{\varphi} g\right)=\int_{0}^{\infty} v(t)\left(\int_{0}^{t} \frac{g(u)}{\varphi(u)} \frac{d u}{u}\right) \frac{d t}{t}=\int_{0}^{\infty} \frac{w(u)}{\varphi(u)} g(u) \frac{d u}{u} .
$$

Therefore $F=L_{*}^{1}(w / \varphi)$.

Note that if $v$ is slowly varying, then we are in a critical case. Indeed, we have $\alpha_{H}=\beta_{H}=0$ and, by the proof of Theorem 5.3, $\alpha_{F}=\beta_{F}=\alpha_{\varphi}$. The last equalities follows also by direct computations because in this case $\omega$ is slowly varying as well. Theorem 5.3 yields that the couple $\left(\rho_{L_{*}^{1}(v)}, \rho_{L_{*}^{1}(w / \varphi)}\right)$ is optimal.

ExAmple 5.5. Let $H=L_{*}^{\infty}(v)$, where $\sup _{1<t<\infty} v(t)<\infty$ and with $\alpha_{H}=\beta_{H}$. Then (5.2) and (3.4) hold. The optimal domain quasi-norm is

$$
\rho_{F}(g)=\sup _{0<t<\infty} v(t) \int_{0}^{t} \frac{g(u)}{\varphi(u)} \frac{d u}{u} .
$$

Again, if $v$ is slowly varying, then we are in a critical case.

The following result will allow us to show another example.

THEOREM 5.6. Let $\rho_{S}$ be a monotone quasi-norm satisfying Minkowski's inequality, with $\alpha_{S}=\beta_{S}=0$ and $\rho_{S}\left(g\left(t^{2}\right)\right) \approx \rho_{S}(g(t))$ for any $g \in \mathbf{M}^{+}$. Let $c, b$ be slowly varying functions on $(0, \infty)$ such that $c(t) \lesssim b(t), c\left(t^{2}\right) \approx c(t)$ and

$$
\begin{aligned}
\int_{0}^{2} g(u) \frac{d u}{u} & \lesssim \rho_{S}(b g), & & g \in \mathbf{M}^{+}, \\
\rho_{S}\left(c \chi_{(1, \infty)}\right) & <\infty, & & \\
\rho_{S}\left(\chi_{(0,2)}(t) c(t) \int_{0}^{t} g(u) \frac{d u}{u}\right) & \lesssim \rho_{S}(b g), & & g \in \mathbf{M}^{+}, \\
\int_{t^{2} / e}^{t} \frac{c(u)}{b(u)} \frac{d u}{u} & \gtrsim 1, & & 0<t<1 .
\end{aligned}
$$

Put

$$
\rho_{F}(g)=\rho_{S}\left(\frac{b g}{\varphi}\right), \quad \rho_{H}(g)=\rho_{S}(c g), \quad g \in \mathbf{M}^{+} .
$$


Then $\left(\rho_{F}, \rho_{H}\right)$ belongs to $\mathcal{N}$, the couple is admissible and $\mathscr{C} H=\mathscr{C} H(F)$.

Proof. The quasi-norm $\rho_{F}$ is monotone and satisfies Minkowski's inequality. Let $0<\varepsilon<\alpha_{\varphi}$. Since $\varphi(1) \leq h_{\varphi}(1 / t) \varphi(t)$, we get for $t>1$

$$
\frac{b(t)}{\varphi(t)} \lesssim t^{-\alpha_{\varphi}+\varepsilon} b(t)=\frac{t^{-\alpha_{\varphi}+\varepsilon} b(t)}{c(t)} c(t) \lesssim c(t)
$$

where we have also used that $b$ and $c$ are slowly varying. Combining this with (5.7) we obtain

$$
\rho\left(\chi_{(1, \infty)}\right)=\rho_{S}\left(\frac{b \chi_{(1, \infty)}}{\varphi}\right) \lesssim \rho_{S}\left(c \chi_{(1, \infty)}\right)<\infty .
$$

The indices of $F$ are equal to those of $\varphi$, so we are in a critical case. Inequality (4.3) is also satisfied because, using (5.6), we get

$$
\int_{0}^{2} \frac{g(u)}{\varphi(u)} \frac{d u}{u} \lesssim \rho_{S}\left(\frac{b g}{\varphi}\right)=\rho_{F}(g) .
$$

Therefore, $\rho_{F} \in \mathscr{N}_{d}$. It is not hard to check that $\rho_{H} \in \mathscr{N}_{t}$. It follows from $c(t) \lesssim b(t)$ that

$$
\rho_{H}\left(\chi_{(0,2)} \frac{g}{\varphi}\right)=\rho_{S}\left(\chi_{(0,2)} \frac{c g}{\varphi}\right) \lesssim \rho_{S}\left(\frac{b g}{\varphi}\right)=\rho_{F}(g) .
$$

Whence $\left(\rho_{F}, \rho_{H}\right) \in \mathcal{N}$. The couple is admissible because, by (5.8),

$$
\rho_{H}\left(\chi_{(0,2)} R_{\varphi} g\right)=\rho_{S}\left(\chi_{(0,2)}(t) c(t) \int_{0}^{t} \frac{g(u)}{\varphi(u)} \frac{d u}{u}\right) \lesssim \rho_{S}\left(\frac{b g}{\varphi}\right)=\rho_{F}(g) .
$$

As for the optimality, it follows from Theorem 5.2 that $\mathscr{C} H(F) \hookrightarrow \mathscr{C} H$. Let us check the converse embedding. According to (3.7), it suffices to show that

$$
\rho_{H(F)}\left(\chi_{(0,2)} g\right) \lesssim \rho_{H}(g), \quad g \in N_{0} .
$$

Given $g$, let $h$ be the function defined by the equality

$$
\frac{b(t) h(t)}{\varphi(t)}=g(\sqrt{4 e t}) c(\sqrt{4 e t}) .
$$

Since $c$ and $b$ are slowly varying functions, we have that $h \in N_{1}$. Using (5.9) 
and the properties of $c$, we have for $0<t<2$

$$
\begin{aligned}
R_{\varphi} h(t) & =\int_{0}^{t} \frac{g(\sqrt{4 e u}) c(\sqrt{4 e u})}{b(u)} \frac{d u}{u} \geq \int_{t^{2} / 4 e}^{t / 2} \frac{g(\sqrt{4 e u}) c(\sqrt{4 e u})}{b(u)} \frac{d u}{u} \\
& \gtrsim g(t) \int_{t^{2} / 4 e}^{t / 2} \frac{c(4 e u)}{b(u)} \frac{d u}{u} \gtrsim g(t) \int_{t^{2} / 4 e}^{t / 2} \frac{c(u)}{b(u)} \frac{d u}{u} \gtrsim g(t) .
\end{aligned}
$$

Consequently,

$$
\begin{aligned}
\rho_{H(F)}\left(\chi_{(0,2)} g\right) & \lesssim \rho_{F}(h)=\rho_{S}\left(\frac{b h}{\varphi}\right)=\rho_{S}(g(\sqrt{4 e t}) c(\sqrt{4 e t})) \\
& \approx \rho_{S}(g(4 e t) c(4 e t)) \lesssim \rho_{H}(g) .
\end{aligned}
$$

In the following example, boundedness of $R_{\varphi}$ can be realized as boundedness of the classical Hardy operator between weighted $L_{q}$ spaces, which is characterized by Muckenhoupt's condition (see [36] or [14, Thm. 2.2.1]).

Example 5.7. Let $1 \leq q \leq \infty$ and $1 / q+1 / q^{\prime}=1$. Put $F=L_{*}^{q}(b / \varphi)$ and $H=L_{*}^{q}(c)$ with $c$ and $b$ being slowly varying functions on $(0, \infty)$, satisfying that $c\left(t^{2}\right) \approx c(t), b(t) \lesssim(1+|\log t|) c(t)$ and

$$
\left(\int_{t}^{\infty} c^{q}(u) \frac{d u}{u}\right)^{1 / q}\left(\int_{0}^{t} b^{-q^{\prime}}(u) \frac{d u}{u}\right)^{1 / q^{\prime}} \lesssim 1, \quad 1 / q+1 / q^{\prime}=1 .
$$

Then $\Lambda(E, F) \hookrightarrow \mathscr{C} H$ and $\mathscr{C} H=\mathscr{C} H(F)$.

Indeed, take $S=L_{*}^{q}$ in Theorem 5.6. It is easy to check that $\rho_{S}\left(g\left(t^{2}\right)\right) \approx$ $\rho_{S}(g(t))$ and $\alpha_{S}=\beta_{S}=0$. Using that

$$
c(t) \lesssim\left(\int_{t}^{\infty} c^{q}(u) \frac{d u}{u}\right)^{1 / q} \quad \text { and } \quad\left(\int_{0}^{t} b^{-q^{\prime}}(u) \frac{d u}{u}\right)^{-1 / q^{\prime}} \lesssim b(t),
$$

we derive from (5.10) that $c(t) \lesssim b(t)$. Moreover, (5.10) also implies (5.6) and (5.7). Namely

$$
\int_{0}^{2} g(u) \frac{d u}{u} \leq\left(\int_{0}^{2}(b(u) g(u))^{q} \frac{d u}{u}\right)^{1 / q}\left(\int_{0}^{2} b(u)^{-q^{\prime}} \frac{d u}{u}\right)^{1 / q^{\prime}} \lesssim \rho_{S}(b g),
$$

and

$$
\rho_{S}\left(c \chi_{(1, \infty)}\right)=\left(\int_{1}^{\infty} c^{q}(u) \frac{d u}{u}\right)^{1 / q}<\infty
$$


Inequality (5.8) is another consequence of (5.10) by Muckenhoupt's result

$$
\rho_{S}\left(\chi_{(0,2)}(t) c(t) \int_{0}^{t} g(u) \frac{d u}{u}\right) \lesssim\left(\int_{0}^{\infty}(b(u) g(u))^{q} \frac{d u}{u}\right)^{1 / q} .
$$

Finally, to check (5.9) note that for $0<t<1$ we have

$$
\int_{t^{2} / e}^{t} \frac{c(u)}{b(u)} \frac{d u}{u} \gtrsim \int_{t^{2} / e}^{t} \frac{1}{1-\log u} \frac{d u}{u}=\log 2 .
$$

ACKNOWLEDGEMENT. It is a pleasure to thank the referee for his/her useful comments which have led to improve the paper.

\section{REFERENCES}

1. Ahmed, I., and Karadzhov, G. E., Optimal embeddings of generalized homogeneous Sobolev spaces on $\mathrm{R}^{n}$, Colloq. Math. 123 (2011), 1-20.

2. Bashir, Z., Cobos, F., and Karadzhov, G. E., Optimal embeddings of Calderón spaces in the super-critical case, C. R. Acad. Bulg. Sci. 65 (2012), 881-890.

3. Bashir, Z., and Karadzhov, G. E., Optimal embeddings of generalized Besov spaces, Eurasian Math. J. 2 (2011), 5-31.

4. Bennett, C., and Sharpley, R., Interpolation of operators, Pure App. Math. 129, Academic Press, New York 1988.

5. Bergh, J., and Löfström, J., Interpolation spaces. An introduction, Grundl. Math. Wiss. 223, Springer, Berlin 1976.

6. Berkolaiko, M. Z., and Ovchinnikov, V. I., Inequalities for entire functions of exponential type in rearrangement invariant spaces, Proc. Steklov Inst. Math. 161 (1983), 3-17.

7. Brudnyi, Yu. A., and Krugljak, N. Ya.,Interpolation functors and interpolation spaces, NorthHolland, Amsterdam 1991.

8. Calderón, A. P., Intermediate spaces and interpolation, the complex method, Studia Math. 24 (1964), 113-190.

9. Cianchi, A., Symmetrization and second order Sobolev inequalities, Ann. Mat. Pura Appl. 183 (2004), 45-77.

10. Cobos, F., and Fernandez, D. L., Hardy-Sobolev spaces and Besov spaces with a function parameter, pp. 158-170 in: Function Spaces and Applications, Lect. Notes Math. 1302, Springer, Berlin 1988.

11. Cobos, F., and Kühn, T., Approximation and entropy numbers in Besov spaces of generalized smoothness, J. Approx. Theory 160 (2009), 56-70.

12. Cwikel, M., and Pustilnik, E., Sobolev type embeddings in the limiting case, J. Fourier Anal. Appl. 4 (1998), 433-446.

13. Cwikel, M., and Pustilnik, E., Weak type interpolation near "endpoint" spaces, J. Funct. Anal. 171 (2000), 235-277.

14. Edmunds, D. E., and Evans, W. D., Hardy operators, function spaces and embeddings, Springer Mon. Math., Springer, Berlin 2004.

15. Edmunds, D. E., Kerman, R., and Pick, L., Optimal Sobolev embeddings involving rearrangement invariant quasinorms, J. Funct. Anal. 170 (2000), 307-355. 
16. Edmunds, D. E., and Triebel, H., Sharp Sobolev embeddings and related Hardy inequalities: the critical case, Math. Nachr. 207 (1999), 79-92.

17. Gogatishvili, A., Neves, J. S., and Opic, B., Optimal embeddings of Bessel-potential-type spaces into generalized Hölder spaces involving $k$-modulus of smoothness, Potential Anal. 32 (2010), 201-228.

18. Gogatishvili, A., and Ovchinnikov, V. I., Interpolation orbits and optimal Sobolev's embeddings, J. Funct. Anal. 253 (2007), 1-17.

19. Gogatishvili, A., and Ovchinnikov, V. I., The optimal embedding for the Calderon type spaces and the J-method spaces, Vestnik Voronežskogo gosudarstvennogo universiteta 1 (2006), 145-151.

20. Goldman, M. L., On embedding for different metrics of Calderon type spaces, Proc. Steklov Inst. Math. 181 (1988), 75-101.

21. Goldman, M. L., Rearrangement invariant envelopes of generalized Besov, Sobolev, and Calderon spaces, Contemporary Math. 424 (2007), 53-81.

22. Goldman, M. L., and Kerman, R. A., On optimal embedding of Calderon spaces and generalized Besov spaces, Proc. Steklov Inst. Math. 243 (2003), 154-184.

23. Gurka, P., and Opic, B., Sharp embeddings of Besov spaces with logarithmic smoothness, Rev. Mat. Complut. 18 (2005), 81-110.

24. Hansson, K., Imbedding theorems of Sobolev type in potential theory, Math. Scand. 45 (1979), 77-102.

25. Haroske, D. D., and Moura, S. D., Continuity envelopes and sharp embeddings in spaces of generalized smoothness, J. Funct. Anal. 254 (2008), 1487-1521.

26. Kerman, R., and Pick, L., Optimal Sobolev imbeddings, Forum Math. 18 (2006), 535-570.

27. Kolyada, V. I., Rearrangements of functions and embedding theorems, Russian Math. Surveys 44 (1989), 73-117.

28. Köthe, G., Topological vector spaces I, Grundl. Math. Wiss. 159, Springer, Berlin 1969.

29. Krein, S. G., Petunin, U. I., and Semenov, E. M., Interpolation of linear operators, Transl. Math. Mon. 54, Amer. Math. Soc., Providence 1982.

30. Maly, J., and Pick, L.,An elementary proof of sharp Sobolev embeddings, Proc. Amer. Math. Soc. 130 (2002), 555-563.

31. Martin, J., and Milman, M., Symmetrization inequalities and Sobolev embeddings, Proc. Amer. Math. Soc. 134 (2006), 2235-2247.

32. Martin, J., and Milman, M., Higher orger symmetrization inequalities and applications, J. Math. Anal. Appl. 330 (2007), 91-113.

33. Martin, J., and Milman, M., Self improving Sobolev-Poincare inequalities, truncation and symmetrization, Potential Anal. 29 (2008), 391-408.

34. Martin, J., Milman, M., and Pustylnik, E., Sobolev inequalities: symmetrization and selfimprovement via truncation, J. Funct. Anal. 252 (2007), 677-695.

35. Milman, M., and Pustylnik, E., On sharp higher order Sobolev embeddings, Comm. Contemp. Math. 6 (2004), 495-511.

36. Muckenhoupt, B., Hardy's inequality with weights, Studia Math. 44 (1972), 31-38.

37. Netrusov, Y. V., Embedding theorems for Besov spaces in symmetric spaces, Notes Sci. Sem. LOMI 159 (1987), 69-102.

38. Nikol'skij, S. M., Approximation of functions of several variables and embedding theorems, Nauka, Moscow 1977.

39. Petrushev, P. P., and Popov, V. A., Rational approximation of real functions, Cambr. Univ. Press, Cambridge 1987.

40. Triebel, H., Interpolation theory, function spaces, differential operators, North-Holland, Amsterdam 1978. 
41. Triebel, H., Theory of function spaces, Mon. Math. 78, Birkhäuser, Basel 1983.

42. Triebel, H., Theory of function spaces III, Mon. Math. 100, Birkhäuser, Basel 2006. GC UNIVERSITY

LAHORE

PAKISTAN

E-mail: ziabashir@gmail.com

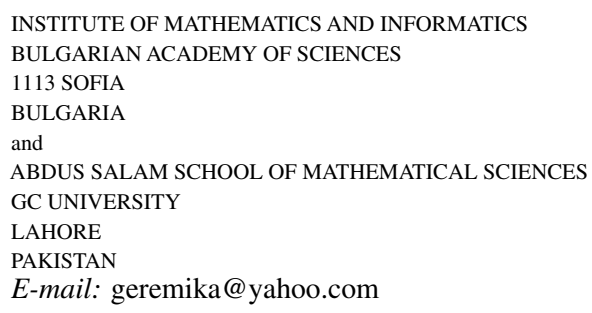

ABDUS SALAM SCHOOL OF MATHEMATICAL SCIENCES
DEPARTAMENTO DE ANÁLISIS MATEMÁTICO, FACULTAD DE MATEMÁTICAS

UNIVERSIDAD COMPLUTENSE DE MADRID

PLAZA DE CIENCIAS 3

28040 MADRID

SPAIN

E-mail: cobos@mat.ucm.es 